\title{
Effect of strain-temperature stress on MOS structure with ultra-thin gate oxide
}

\author{
Chia-Nan Lin, Yi-Lin Yang, Wei-Ting Chen, Shang-Chih Lin, Kai-Chieh Chuang, Jenn-Gwo Hwu* \\ Graduate Institute of Electronics Engineering, Department of Electrical Engineering, National Taiwan University, Room 446, Taipei 106, Taiwan, ROC
}

\section{A R T I C L E I N F O}

Article history:

Received 26 February 2008

Received in revised form 23 May 2008

Accepted 17 June 2008

Available online 22 June 2008

\section{Keywords:}

Oxides

Strain-temperature

Interface

Breakdown

Leakage

\begin{abstract}
A B S T R A C T
A method called strain-temperature stress was adopted in this work to improve the quality of ultra-thin oxide on both $\operatorname{MOS}(\mathrm{p})$ and $\operatorname{MOS}(\mathrm{n})$ capacitors. MOS structures were baked at $100{ }^{\circ} \mathrm{C}$ under externally applied mechanical stress. Reduced gate leakage current, reduced interface trap density $\left(D_{\text {it }}\right)$, and improved time-dependent-dielectric-breakdown (TDDB) characteristics were observed after tensile-temperature stress treatment without increasing the oxide thickness. On the contrary, compressive-temperature stress resulted in a degraded performance of MOS capacitors. Consequently, the tensile-temperature stress method is suggested as a possible technique to enhance the ultra-thin oxide quality of MOS structure.
\end{abstract}

(c) 2008 Elsevier B.V. All rights reserved.

\section{Introduction}

Silicon dioxide $\left(\mathrm{SiO}_{2}\right)$ has been used as the gate dielectric of metal-oxide-semiconductor field-effect transistors (MOSFETs) for over half a century due to its excellent stability, uniformity and simple fabrication process. As MOS devices are scaled down to the deep-submicrometer region, the thickness of $\mathrm{SiO}_{2}$ should also be scaled down to achieve better performance. Based on the International Technology Roadmap for Semiconductor (ITRS), equivalent oxide thickness (EOT) should be scaled to $0.6 \mathrm{~nm}$ in 2013 [1]. The issue of thermal stress is of special concern as the power density increases with the scaling down of device. Since the thermal expansion coefficients between packaging material and enclosed chip are generally different, the mechanical stress sustained by the two different materials exists. The induced package-related stress may result in devices degradation, circuit malfunction and chip breakup. Device degradation due to the thermal or mechanical stress during operation is therefore of concern [2-4]. However, on the contrary, strained silicon has positive effect on channel mobility. The improvement in carrier mobility has been intensely studied by introducing strain in the channel region, such as strained-Si on SiGe substrate $[5,6]$.

In this study, our works focus on the mechanical stress effect on MOS structures at elevated temperatures. It is well known that the atomic bonding in devices may change under external stresses such as thermal stress, mechanical stress and electrical stress. In order to simulate the situation that devices sustain mechanical and temperature stresses simultaneously, an effective method called

\footnotetext{
* Corresponding author. Tel.: +886 2 23635251x446; fax: +886 223671909

E-mail address: hwu@cc.ee.ntu.edu.tw (J.-G. Hwu).
}

strain-temperature stress was proposed [7]. Both the electrical characteristics of MOS structures after receiving various mechanical stresses under elevated temperatures and the effects of thermal-mechanical stress on oxide reliability were investigated.

\section{Experimental}

Both boron- and phosphorous-doped 3-inch silicon wafers with a resistivity of $1-10 \Omega \mathrm{cm}$ were used as the substrates of MOS capacitors. After standard RCA clean, the oxides were grown either by rapid thermal process (RTP) or anodization. The oxide thicknesses are around $25 \AA$ for p-type and $20 \AA$ for n-type. After front Al evaporation, capacitors with a $150 \mu \mathrm{m} \times 150 \mu \mathrm{m}$ area were formed by conventional photolithography. The back oxide is then removed by using buffered oxide etchant (BOE) and then $\mathrm{Al}$ is evaporated as the back contact.

After the device fabrication, a quartz holder was used to strain the wafer as shown in Fig. 1a. The differences between tensileand compressive-stress were sketched as shown in Fig. 1b and c, respectively. For strain-temperature treatments, samples with various stress conditions were baked at the temperature of $100^{\circ} \mathrm{C}$.

\section{Result and discussion}

As the strain-temperature stress was applied, both the thermal and mechanical stresses take place simultaneously. In order to make sure that the variation of electrical characteristics was caused by the strain effect, samples without any mechanical stress were baked at the same time. The $J-V$ curves of both p- and n-type samples baked at $150{ }^{\circ} \mathrm{C}$ were plotted as shown in Fig. 2a and b, respectively. It could be observed that there is nearly no change 

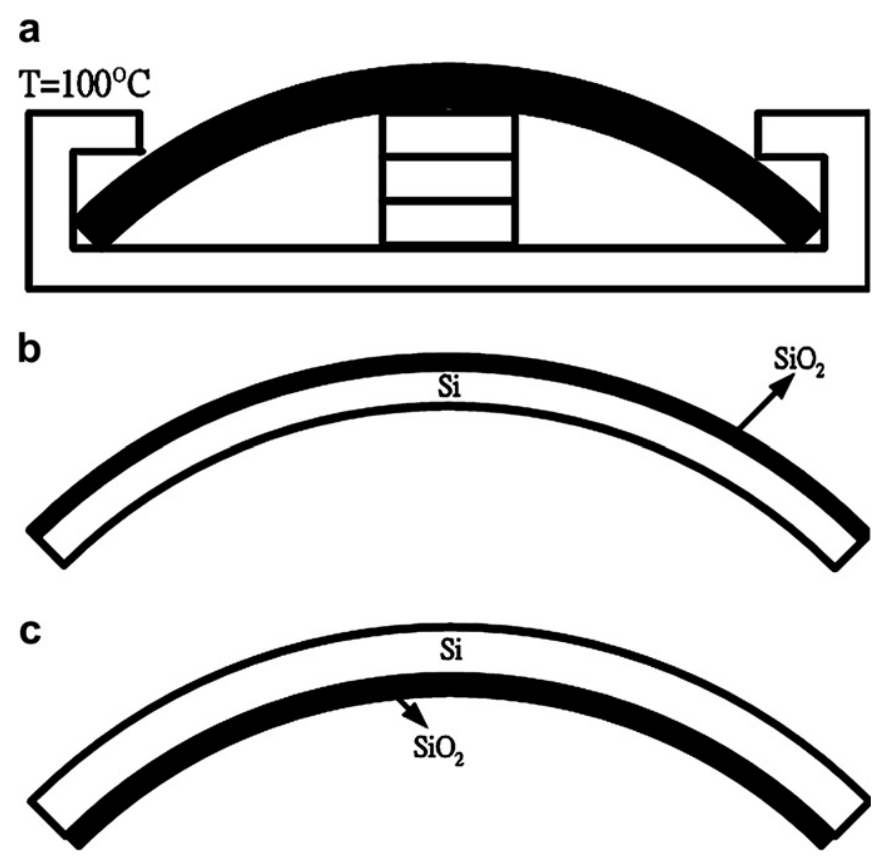

Fig. 1. Front views of (a) strain-temperature experimental setup, (b) tensile-stress sample, and (c) compressive-stress sample.

in $J-V$ curves even after baking at $150^{\circ} \mathrm{C}$ for $30 \mathrm{~min}$. As a consequence, the variations observed in the following experimental results could be considered as the influence of additional mechanical stress. In order to discuss the strain-temperature phenomenon in detail, four sections are discussed.

\subsection{J-V characteristic in p-type samples}

Fig. 3 shows the comparison of substrate injection current variations at $2 \mathrm{~V}$ for non-stress, compressive-stress and tensile-stress MOS(p) samples. After baking at $100^{\circ} \mathrm{C}$ for $5 \mathrm{~min}$, the devices were measured immediately and the measured data were named as "baked 1". The devices were then stayed at room temperature for 1 week and the measured data were named as "stable 1 ". The stress cycle was then repeated and the data named "baked 2" and "stable 2" could be measured subsequently. For non-stress samples, it was found that the substrate injection current increased due to the thermal stress effect but got recovery after 1 week at room temperature. For tensile-stress samples, the substrate injection current in stable state is smaller than that of initial state. On the contrary, the substrate injection current increased for the compressive stressed samples. The difference in current variations could be explained by analyzing the components in substrate injection current. It is known that diffusion current $\left(J_{\text {diff }}\right)$, depletion region generation current $\left(J_{\mathrm{rg}}\right)$, and surface state generation current $\left(J_{\text {st }}\right)$ are the three main components of substrate injection current [8]. In general, $J_{\text {diff }}$ is small when the temperature is lower than $160{ }^{\circ} \mathrm{C}$. On the other hand, $J_{\mathrm{rg}}$ is stable because this component is strongly dependent on the bulk trap density in silicon. As the result, the component of $J_{\text {st }}$, which is strongly dependent on interface trap density $\left(D_{\text {it }}\right)$, affects the substrate injection current in a big way. It can be concluded that the substrate injection current is strongly affected by $D_{\mathrm{it}}$. In other words, the current variations might be mainly due to the changes of $D_{\text {it. }}$. The results of calculated interface trap densities will be illustrated in session C. It is known that the lattice constants of $\mathrm{SiO}_{2}$ and $\mathrm{Si}$ are different. The mismatch in lattice constant may cause a large number of dangling bonds. To re-build the atomic bonding, the Si wafer needs enough energy which may be provided by elevated temperatures. Here, we sug-
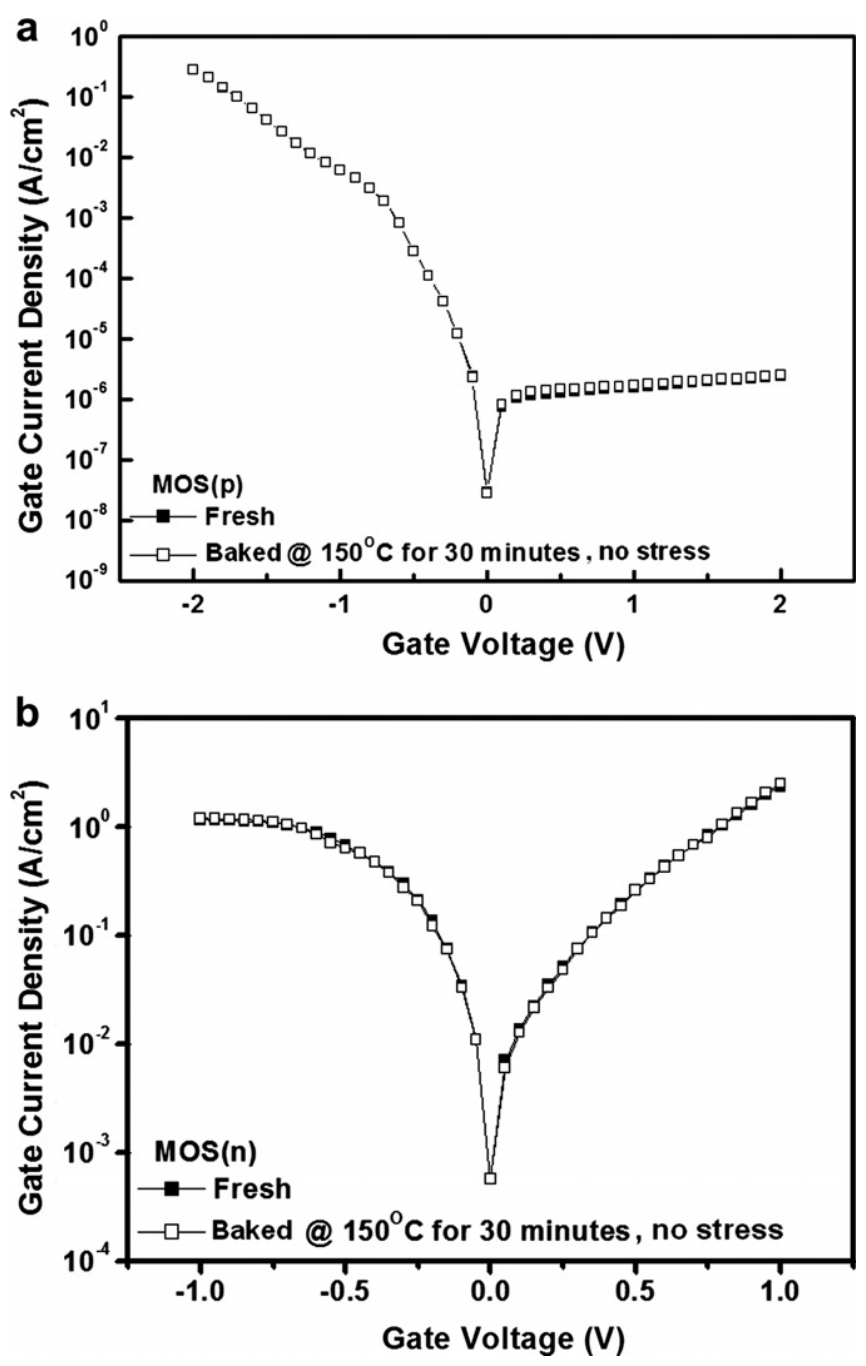

Fig. 2. The $J-V$ curves of (a) $\operatorname{MOS}(\mathrm{p}$ ) and (b) $\operatorname{MOS}(\mathrm{n}$ ) samples before and after being baked at $150{ }^{\circ} \mathrm{C}$ for $30 \mathrm{~min}$ without stress.

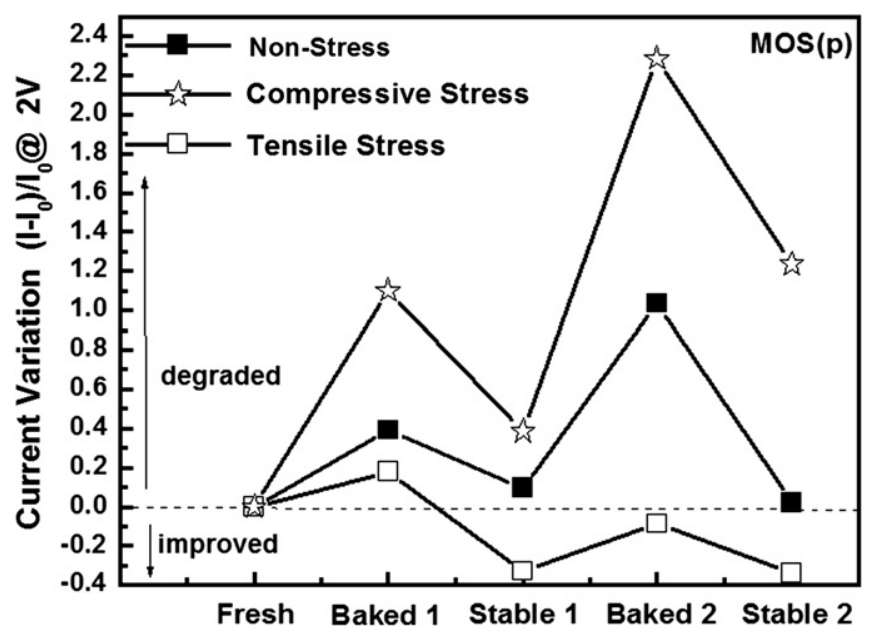

Fig. 3. Comparison of current variations for non-stress, compressive-stress, and tensile-stress $\operatorname{MOS}(\mathrm{p})$ samples.

gest a possible mechanism. $\mathrm{SiO}_{2}$ is known to have larger lattice constant than $\mathrm{Si}$, and the lattice constant mismatch causes the existence of dangling bonds at interface. As the tensile-stress was applied, the lattice constant of $\mathrm{Si}$ near $\mathrm{Si} / \mathrm{SiO}_{2}$ interface would be 
extended because the second order lattice constants are related by stresses and elastic strains [9]. As a result, the lattice constant difference between $\mathrm{Si}$ and $\mathrm{SiO}_{2}$ would be reduced. As the thermal stress was applied at the same time, the dangling bonds would get energy to re-build themselves whereas the compressive-temperature stress would increase the difference of lattice constant and thus increases the $D_{\mathrm{it}}$. It is therefore suggested that the $D_{\mathrm{it}}$ was reduced after tensile-temperature stress but increased after compressive-temperature stress.

Fig. 4 shows the comparison of current variations for four various tensile-temperature stress conditions of $\operatorname{MOS}(\mathrm{p})$ samples. The stress value can be estimated according to the following equation [10-11].

$\sigma=\frac{12 E y t}{4 a^{2}-3 L^{2}}$

where $E$ is the Young's modulus ( $E=130 \mathrm{GPa}$ for (100) silicon orientation), $y$ is the total thickness of the bending stacks $(y=1.52 \times$ $10^{-3} \mathrm{~m}$ for the bending stacks), $t$ is the total thickness of the wafer $\left(t=3.8 \times 10^{-4} \mathrm{~m}\right), L$ is the diameter of the wafer $\left(L=7.62 \times 10^{-2} \mathrm{~m}\right)$ and $\mathrm{a}$ is equal to $L / 4$. The stress values for light, moderate, and strong tensile-stress are 42.3, 56.4, and $70.5 \mathrm{MPa}$, respectively. For the long-time tensile-stress sample, it was stressed with $56.4 \mathrm{MPa}$ and baked for $30 \mathrm{~min}$ while the others were baked for $5 \mathrm{~min}$. It shows that the device under strong tensile-stress reveals better $\mathrm{Si} / \mathrm{SiO}_{2}$ interface quality than the moderate and light tensile stressed samples. On the other hand, although the long-time tensile-stress sample was stressed with moderate stress value, it exhibits the best $\mathrm{Si} / \mathrm{SiO}_{2}$ interface quality. In short, both applying larger tensile-stress or increasing the baking time could improve the interface quality.

\subsection{J-V characteristic in n-type samples}

In addition to $\operatorname{MOS}(\mathrm{p})$ samples, it is interesting to discover the effects of strain-temperature stress on $\operatorname{MOS}(n)$ samples. From the prior experimental results, it is known that tensile-temperature stress enhances the performance of ultra-thin oxide, and the long-time tensile-stress shows the best improvement in comparison with the others. Therefore, the long-time tensile-stress was utilized in this experiment.

The cumulative distributions of gate current density for anodization (ANO) oxides and rapid thermal oxidation (RTO) oxides were shown in Fig. 5 and Fig. 6, respectively. The insets of Fig. 5 and Fig. 6 show the typical $J-V$ curves before and after tensile-tem-

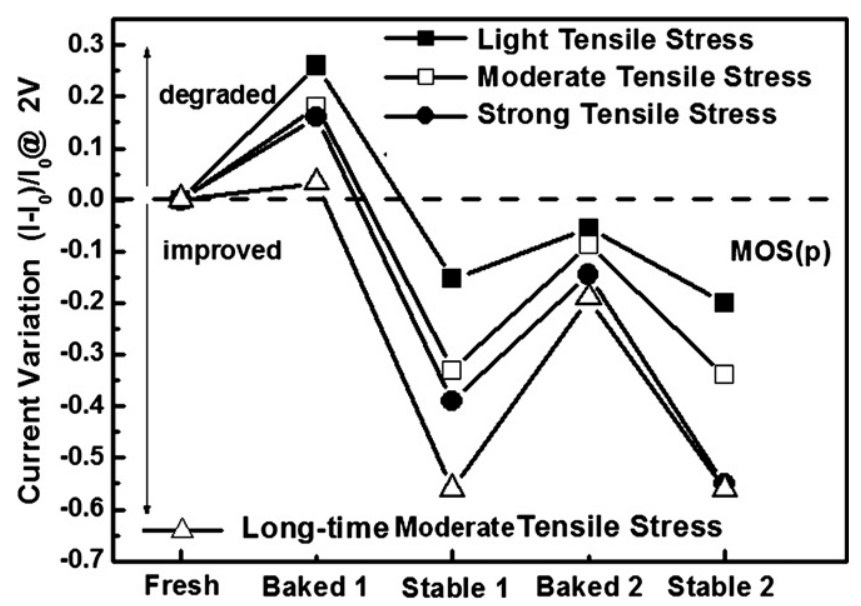

Fig. 4. Comparison of current variations for light, moderate, strong and long-time tensile-stress $\operatorname{MOS}(\mathrm{p})$ samples.

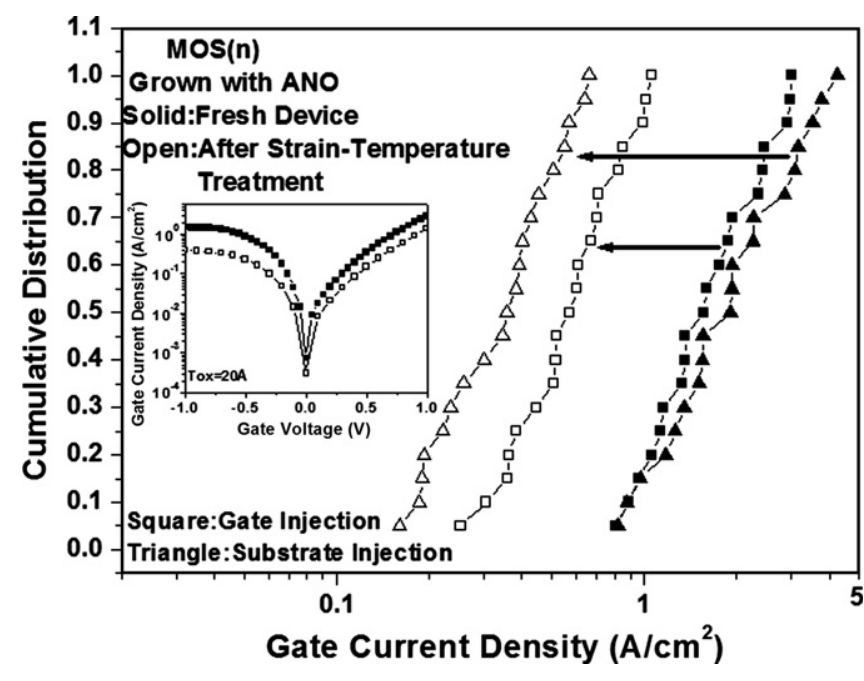

Fig. 5. Cumulative distributions of gate injection and substrate injection currents for MOS(n) devices with ANO oxides. The inset shows the typical $J-V$ curves of $\operatorname{MOS}(\mathrm{n})$ sample before and after tensile-temperature stress treatment.

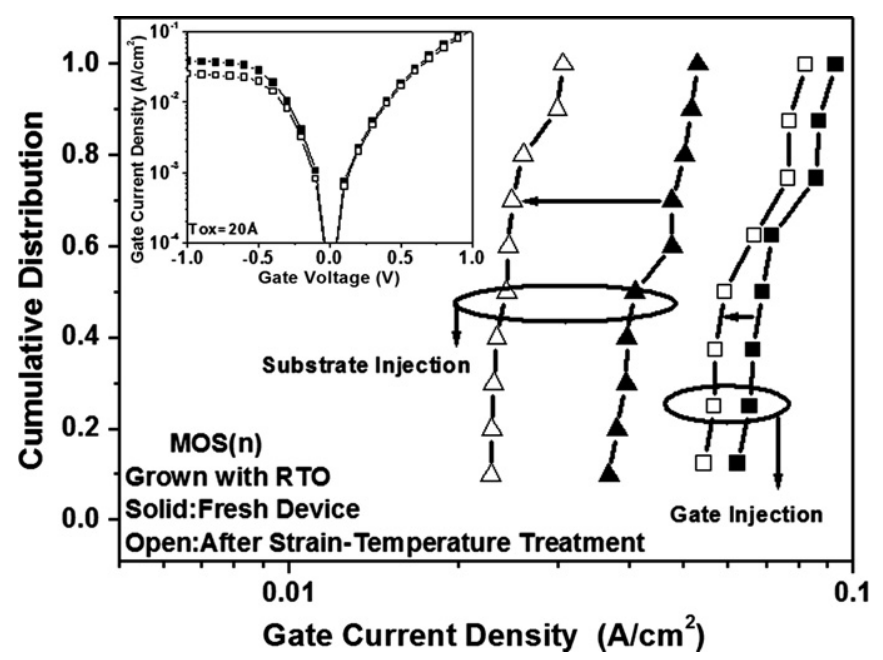

Fig. 6. Cumulative distributions of gate injection and substrate injection currents for $\operatorname{MOS}(n)$ devices with RTO oxides. The inset shows the typical $J-V$ curves of $\operatorname{MOS}(\mathrm{n})$ sample before and after tensile-temperature stress treatment.

perature stress. For the RTO MOS(n) devices, just like the MOS(p) devices, the gate injection currents are almost the same before and after tensile-temperature stress treatment. On the contrary, the gate injection currents reduced apparently in the ANO MOS(n) devices as shown in Fig. 5. It is supposed that during the anodization process the negative ions were attracted to the wafer by the positive electric field and thus would create hidden defects in the oxide. The hidden defects might introduce the increase in gate leakage current. As the tensile-temperature stress was applied, the above defects would be eliminated thus reduces the gate injection current density. However, for both the RTO and ANO samples, an apparent reduction in substrate injection current due to tensiletemperature stress could be observed. The results are also believed due to the improvement in interface performance as stated above.

\subsection{Extracted $D_{i t}$ for both $n$ - and p-type devices}

The extracted $D_{\text {it }}$ at flatband condition of RTO oxides for both $\operatorname{MOS}(\mathrm{p})$ and $\operatorname{MOS}(\mathrm{n})$ devices before and after tensile-temperature stress treatment were plotted in Fig. 7. The $D_{\text {it }}$ is extracted by using 


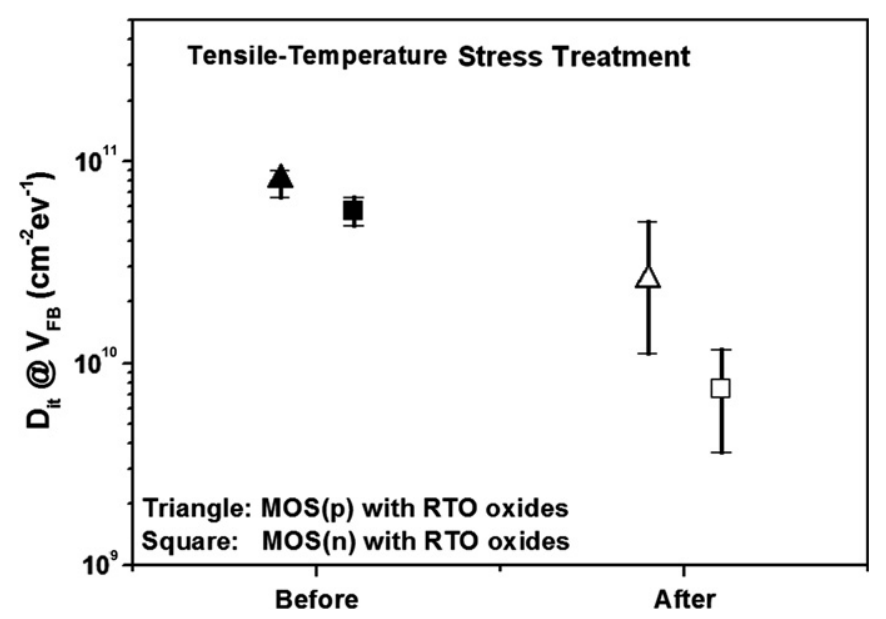

Fig. 7. Extracted $D_{\text {it }}$ at flatband voltage for $\operatorname{MOS}(\mathrm{p})$ and $\operatorname{MOS}(\mathrm{n})$ samples before and after tensile-temperature stress treatment.

two frequencies $C-V$ method [12], and the frequencies used here were $1 \mathrm{M}$ and $10 \mathrm{kHz}$. For both $\operatorname{MOS}(\mathrm{p})$ and $\operatorname{MOS}(\mathrm{n})$ devices, it could be observed that $D_{\text {it }}$ reduces apparently after the tensiletemperature stress treatment. This observation is consistent with the prior suggestion that the tensile-temperature stress would re-build the dangling bonds at $\mathrm{Si} / \mathrm{SiO}_{2}$ interface and therefore reduce the $D_{\text {it }}$.

\subsection{Reliability of p-type strain-temperature stressed samples}

Fig. 8 and Fig. 9 show the cumulative failure distribution of time-to-breakdown $t_{\mathrm{BD}}$ for compressive-temperature, tensile-temperature, and control $\operatorname{MOS}(\mathrm{p})$ samples under constant voltage $(-17.6 \mathrm{MV} / \mathrm{cm})$ stress and constant current $\left(-300 \mathrm{~A} / \mathrm{cm}^{2}\right)$ stress, respectively. As the stress was applied, the electrons gain enough kinetic energy and incur collisions with atoms in oxides thus induce the traps. The increased traps may connect to a leaky path and then eventually cause the oxide breakdown. As the gate oxide breakdowns, the gate current increases and the gate field decreases abruptly. The inset of Fig. 8 reveals that the tensile-temperature stressed sample presents the best ability to prevent breakdown, while the compressive-temperature stressed sample exhibits the worst. In the same way, the inset of Fig. 9 also shows that the ten-

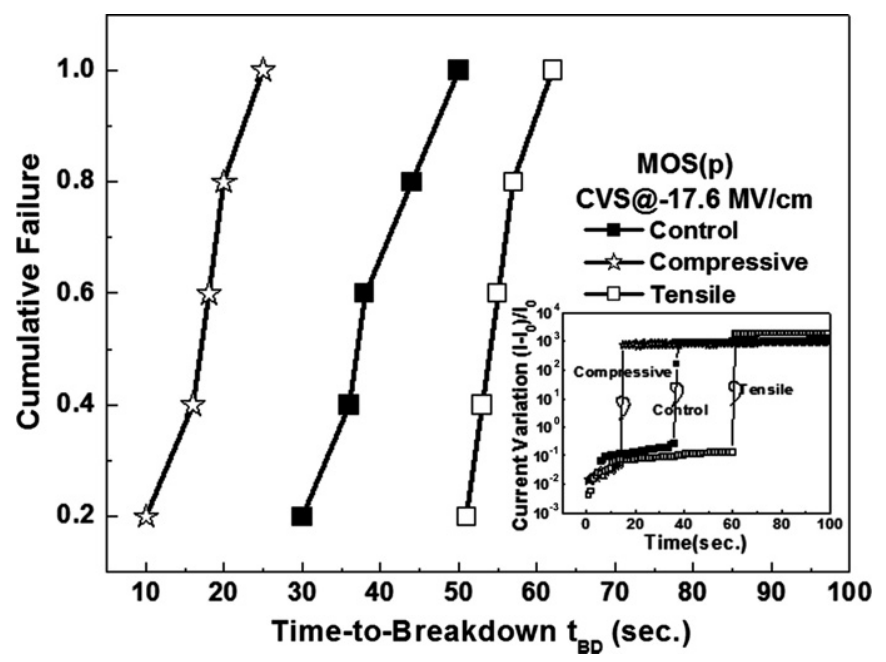

Fig. 8. Cumulative distribution of time-to-breakdown $t_{\mathrm{BD}}$ for $\operatorname{MOS}(\mathrm{p})$ samples after constant voltage stress with various strain-temperature conditions. The inset shows the typical current variations versus stress time.

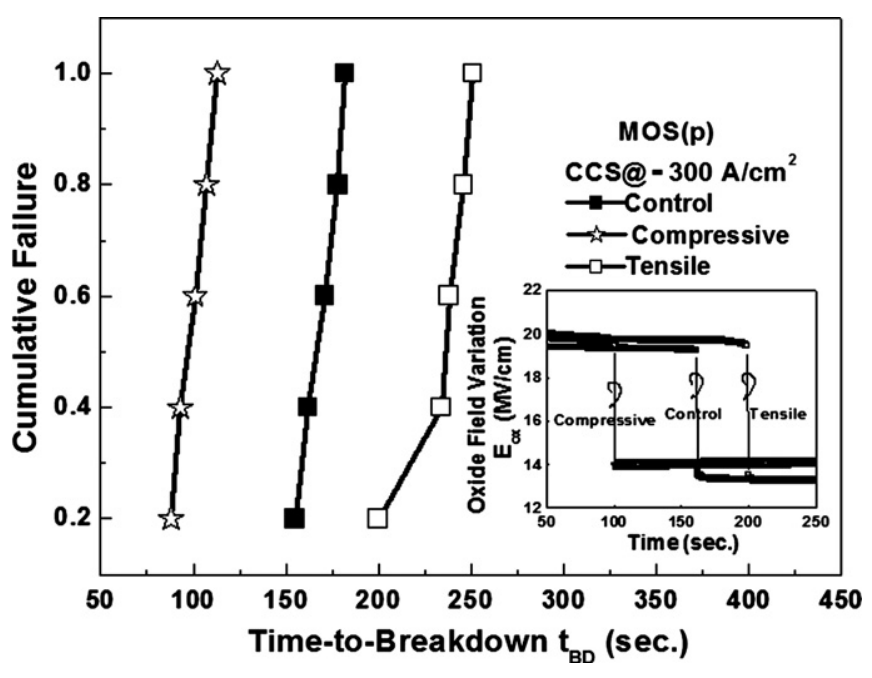

Fig. 9. Cumulative distribution of time-to-breakdown $t_{\mathrm{BD}}$ for $\operatorname{MOS}(\mathrm{p})$ samples after constant current stress with various strain-temperature conditions. The inset shows the typical electric field variations versus stress time.

sile-temperature stressed sample performs the best reliability in constant current $t_{\mathrm{BD}}$ characteristics. Moreover, both the variations in the insets of Fig. 5 and Fig. 6 show barrier lowering which can be accounted for effective hole trapping. Also, it could be observed that the effective hole traps generated in tensile-temperature stressed sample are less than those in control and compressivetemperature stressed samples. It is known that the breakdown characteristics are related to interfacial property and trapping behavior [13-15]. Both the mechanisms, such as filling of intrinsic traps and stress-induced traps generation, had been proposed to explain the oxide degradation during high field stressing. The interface trap generation is strongly related to the $\mathrm{Si} / \mathrm{SiO}_{2}$ interfacial bonding as well. The accelerated electrons can break the weak bonds and cause damage at the interfaces. Since the tensile-temperature stress treatment is suggested to be able to enhance the interfacial bonding and reduce $D_{\text {it }}$, it is rational to observe that the tensile-temperature samples demonstrate improved interfacial property. The stronger interfacial strained bonds in tensile-temperature stressed samples prevent the damage from hot electrons and reinforce the breakdown endurance. In addition, the improvement of $\operatorname{MOS}(\mathrm{n})$ in time-zero dielectric reliability tests is also observed. It is effective in improving the oxide reliability by utilizing tensile-temperature stress.

\section{Conclusion}

In this work, the effect of strain-temperature stress on MOS structure is explored. The experimental results show that the tensile-temperature stressed devices exhibit the improved performance in electrical characteristics with respect to compressive and control samples. The tensile-temperature stressed oxides exhibit not only the reduced substrate injection current but also the improved endurance of breakdown. It is believed that samples after packaging and operated under certain temperature should sustain strain-temperature effect inevitably. The effect of straintemperature stress is important to MOS capacitors with ultra-thin oxides.

\section{Acknowledgement}

The authors want to thank the National Science Council, ROC, for supporting this work under Contract No. NSC 96-2628-E-002246-MY3. 


\section{References}

[1] International Technology Roadmap for Semiconductor, Semiconductor Industry Association, 2004.

[2] N. Zhu, J.D. Van Wyk, Z.X. Liang, in: Power Electronics Specialists Conference, Aachen, Germany, June 20-25, 2004, p. 4503.

[3] A. Hamada, T. Furusawa, N. Satio, E. Takeda, IEEE Trans. ED 38 (1991) 895

[4] T.C. Yang, K.C. Saraswat, IEEE Trans. ED 47 (2000) 746.

[5] J. Welser, J.L. Hoyt, J.F. Gibbons, IEEE Trans. ED Lett. 15 (1994) 100.

[6] K. Rim, J.L. Hoyt, J.F. Gibbons, IEEE Trans. ED 47 (2000) 1406.

[7] C.W. Tung, Y.L. Yang, J.G. Hwu, IEEE Trans. ED 53 (2006) 1736.
8] C.C. Hong, J.G. Hwu, Appl. Phys. Lett. 79 (2001) 3797.

[9] C. Kittel, Introduction to Solid State Physics, fourth ed., New York, Wiley, 1971 (Chapter 4)

[10] C. Gallon, G. Reimbold, G. Ghibaudo, R.A. Bianchi, R. Gwoziecki, S. Orain, E. Robilliart, C. Raynaud, H. Dansas, IEEE Trans. ED 51 (2004) 1254.

[11] K.J. Yang, C. Hu, IEEE Trans. ED 46 (1999) 1500.

[12] S.W. Huang, J.G. Hwu, IEEE Trans. ED 50 (2003) 1658.

[13] K.J. Yang, Y.C. King, C. Hu, in: Technical Digest VLSI Symposium, 1999, p. 77.

[14] M.Y. Doghish, F.D. Ho, IEEE Trans. ED 39 (1992) 2771

[15] E. Rosenbaum, L.F. Register, IEEE Trans. ED 44 (1997) 317. 